Int. J. Dev. Biol. 64: 21-28 (2020)

https://doi.org/10.1387/ijdb.190290mj

\title{
Caulonema differentiation in Funaria protonema
}

\author{
MAN-MOHAN JOHRI* \\ Tata Institute of Fundamental Research, Department of Biological Sciences, Mumbai, India
}

\begin{abstract}
The strategies and experimental approaches that led the author to demonstrate the role of auxin in caulonema differentiation in the protonema of the moss Funaria hygrometrica are discussed. In stationary suspension cultures, the status of cell differentiation is regulated by inoculum cell density and auxin level. At low inoculum cell densities, 2-5 $\mu \mathrm{M}$ indole acetic acid (IAA) led to the differentiation of $65-70 \%$ caulonema filaments in 5-6 days. Caulonema can also differentiate in auxin-free medium if buffered at pH 5.0 after a lag of $6 \pm 1$ days. The duration of lag can be manipulated and the cells are capable of responding to auxin at a higher level (3-10 $\mu \mathrm{M})$ and produce about $20 \%$ caulonema after 3 days. This responsiveness or sensitivity to auxin can be enhanced further by growing cells in a nutrient-limited medium buffered at pH 5.0. In this medium, addition of $3 \mu \mathrm{M}$ IAA led to the differentiation of $75-80 \%$ caulonema and rhizoids within 3 to 4 days. Work done in other laboratories has shown that auxin promotes caulonema differentiation in the moss Physcomitrella patens by positively regulating two basic helix-loop-helix type of transcription factor genes namely root hair defective six-like1 (PpRSL1) and PpRSL2 (Jang and Dolan 2011, New Phytologist 192: 319-327).
\end{abstract}

KEY WORDS: Funaria, moss protonema, Caulonema differentiation, auxin

\section{Introduction}

The protonema in many mosses such as Funaria hygrometrica and Physcomitrella patens represents the filamentous stage of the gametophytic generation and has been widely used to understand the regulation of cell differentiation by phytohormones. It can be readily-grown on a chemically defined solid medium and the differentiation of various cell types followed and quantified. The protonema development in $F$. hygrometrica involves two distinct stages, the chloronema and the caulonema (reviewed in Johri, 1974; 1975). The spores germinate to form the multicellular and cylindrical chloronema filaments and as the development progresses, the caulonema filaments differentiate; these two types of filaments can be easily distinguished on the basis of cellular size and morphology, number and size of chloroplasts in each cell and the orientation of cross wall or septum (Fig. 1). The chloronema marks the default state of differentiation. Upon culturing any part of the protonema or leafy gametophore or diploid sporophytic tissue such as immature green setae, a new protonema regenerates. Protonema developing from the setae are diploid and grow slower as compared to the haploid ones. Upon sub-culture, all cell types first dedifferentiate, undergo reprogramming to form a chloronema apical cell and only later caulonema filaments differentiate. Because of their ability to self-regenerate, the apical cells have also been called as stem cells. The genes conferring the identity of various types of stem cells have been investigated by Hasebe's group (Ishikawa et al., 2011; Kofuji and Hasebe, 2014; Sato et al., 2017). The bud initials are formed only on caulonema cells and their formation is enhanced by biologically active cytokinins. Bud initials ultimately develop into the familiar 3-dimensional leafy moss plants called as gametophores. Auxins initiate rhizoid development in the thalli of liverworts and at the base of the moss plants (Ashton et al., 1979; Kaul et al., 1962; Sakikabara et al., 2003).

Considering the simplicity, I was attracted to this developmental system and started working on moss in 1970 while at Tata Institute of Fundamental Research (TIFR) in Mumbai. I was first exposed to the moss system during 1965-68 when I was a Research Associate at the Plant Research Laboratory (PRL, East Lansing, Mich., USA). At PRL, Hans Brandes in the laboratory of Hans Kende, was investigating cytokinin response in Funaria protonema (Brandes and Kende, 1968). He used to spray a suitably-diluted suspension of moss spores on the solidified medium overlaid with cellophane paper using $15 \mathrm{~cm}$ diameter Petri dishes. After a few days the spores germinated and one could follow the growth of

Abbreviations used in this paper: IAA, indole acetic acid; NAA, naphthalene acetic acid; TIFR, Tata Institute of Fundamental Research (TIFR).

*Address correspondence to: Prof. M.M. Johri. 32 New Cosmos, Juhu-Versova Link Road, Andheri (W), Mumbai 400053 , India. Tel: +91 9930564129.
E-mail: mjohri06915@yahoo.co.in - (iD) https://orcid.org/0000-0003-3131-2739

Submitted: 2 September, 2019; Accepted: 28 October, 2019.

ISSN: Online 1696-3547, Print 0214-6282 


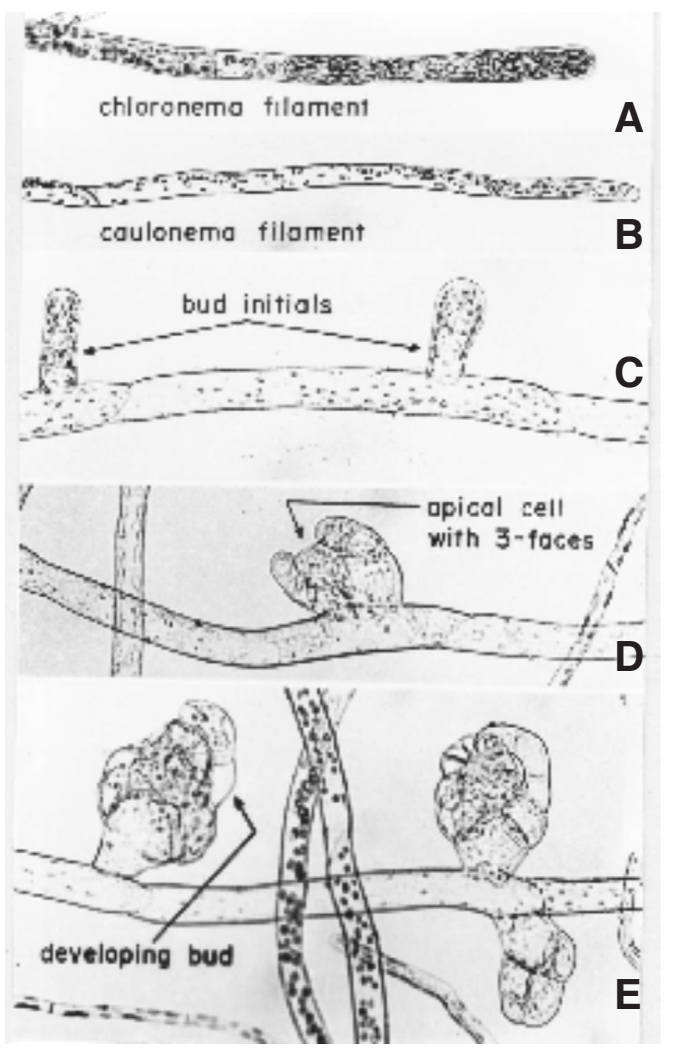

Fig. 1. Cell types in Funaria protonema. (A,B)Typical chloronema and caulonema filaments. (C) Bud initials $24 \mathrm{~h}$ after $1 \mu \mathrm{M}$ kinetin treatment. (D,E) Developing buds 50 and $76 \mathrm{~h}$ after kinetin treatment. Originally published in Johri, MM (1988). Development and differentiation in plants. Bioscience Reports. 8: 553-564.

protonema and formation of various cell types. During those years, the suspension cultures were not used because caulonema did not differentiate in the liquid medium. The gentle shaking of the cultures was also not helpful. While at TIFR, the moss system looked sufficiently interesting to me and I realized that the lack of caulonema differentiation in liquid medium, really offers an advantage and also an opportunity to investigate the regulation of its differentiation. This was the starting point of my interest in the mosses from the developmental biology angle.

\section{Isolation of pipettable cell lines}

Right from the beginning I wanted to develop the moss system so that it is amenable to microbiological techniques. Ideally, one should be able to serially dilute the protonemal cell suspensions, plate it on the solid medium and also grow in the liquid medium on a preparative scale. The protonema grew quite well in continuous light in the liquid MMG (minimal medium with glucose, contains $4.2 \mathrm{mM}$ calcium) but such cultures were not pipettable (imagine a situation where one is trying to pipette greenish cotton-like mass suspended in liquid medium). Here we thought of a very simple strategy to improve pipettability. Since lot of calcium, typically in $\mathrm{mM}$ range, is associated with the plant cells walls and far lower levels, typically in $\mathrm{nM}$ to $\mathrm{pM}$ range, are actually needed for the cellular functions, it was reasoned that by withholding or reducing the calcium in the medium, defective cell walls may be formed which may cause dissociation of filaments leading to pipettable cell suspensions. The protonema was therefore grown for several passages in the LCM (low-calcium medium, contains $0.21 \mathrm{mM}$ calcium); where the calcium levels were reduced to a level such that the growth rate was not affected. After several passages, it was found that the by simply swirling the flask gently, the chloronema filaments in the stationary phase underwent dissociation and could be easily dispersed. Such suspensions comprised cellular aggregates of short, multicellular filaments of various sizes (free cells to 15-celled) and could be pipetted, serially diluted and plated.

It was fortuitous that a strategy of reducing the calcium levels was employed initially and much later it was found that the dissociation was really due to an entirely different reason (Johri, 2004). The low level of calcium acted as a nutritional stress and limited the multiplication of plastids and this stress led to the formation of yet another cell type, namely the tmema cells (Bopp et al., 1991). The tmema cells are normally produced by protonema older than 25 days in response to ethylene (Rohwer and Bopp, 1985). Filaments with tmema cells were quite fragile and as already stated upon manually swirling the flask, the stationary phase chloronema filaments dispersed easily into cellular aggregates consisting of $1-15$ cells. The larger aggregates ( $>6$ celled filaments) represented only a small fraction and most of the aggregates tended to be 1-5 celled. Each cell or cellular aggregate regenerated into protonema upon culture on solid or liquid medium with a normal level of nutrients (MM or MMG medium). To ensure that the cells used as inoculum are actively growing, the stationary phase dissociated cell suspensions were pre-cultured for 24 hours in fresh LCM and then used. Thus, essentially speaking, the inoculum was maintained by repeatedly sub-culture in LCM and all experiments were carried out using MMG (or MM). This strategy was used successfully for over 30 years during 1970-2002, in my laboratory at TIFR.

\section{Caulonema differentiation in suspension cultures}

The first clue about the regulation of caulonema differentiation emerged as follows. In order to isolate mutants auxotrophic for amino acids, purines and pyrimidines, the spores mutagenized with UV were plated on the MM supplemented with casamino acids and yeast extract. On this medium, the spores germinated but further development remained inhibited and ultimately no protonema developed. Further analysis showed that histidine and tyrosine were strongly inhibitory for protonema growth and tryptophan though slightly inhibitory, enhanced caulonema formation as compared to control protonema (reviewed in Johri, 1975). This observation suggested that IAA could be involved in cell differentiation and was followed up using the liquid cultures in which as already stated no caulonema are formed. The initiation of primary caulonema ( $1^{\circ}$ caulonema) was strongly dependent on the inoculum cell density and $6-10 \%$ differentiated at cell densities below $0.05 \mathrm{mg} /$ $\mathrm{ml}$. Caulonema proportion increased to $20-25 \%$ when cells were grown in MM containing 25-100 $\mu \mathrm{M}$ tryptophan. This observation was followed up by studying the effect of auxins. Inoculum size and auxin levels were found to be extremely important parameters in liquid cultures. At $5 \mu \mathrm{M} \mathrm{IAA}$, a 5- or 6-day-old culture contained predominantly caulonema (70-80\%). $\alpha$-NAAwas also equally effective while $\beta$-NAA and 2,4-D (2,4-dichlorophenoxyaceticacid) were completely inactive. Exogenous auxin evoked two responses; at low levels (apparent $K m 0.1 \mu \mathrm{M}$ ) it inhibited the formation of secondary 
$\left(2^{\circ}\right)$ chloronema and at a slightly higher level (apparent $\mathrm{Km} 0.4$ $\mu \mathrm{M})$ it increased the production of $2^{\circ}$ and higher order caulonema. By manipulating the level of exogenous auxin and inoculum size, cultures containing $>65-70 \%$ caulonema were readily obtained routinely (Johri and Desai, 1973). As the $2^{\circ}$ caulonema are formed at IAA concentrations higher than those inhibiting chloronema, both responses occur together in liquid culture. Several other auxins have also been tested and ethyl ester of IAA is slightly less active then IAA in enhancing caulonema (reviewed in Johri and D'Souza, 1990).

By raising auxin level to 2.5-5.0 $\mu \mathrm{M}$, caulonema differentiation is observed even at higher cell densities of $0.5-1 \mathrm{mg} / \mathrm{ml}$ (Johri, 1975). Since at these high cell densities, formation of all caulonema was strictly auxin-dependent, we can conclude that the basal level of caulonema formation at low densities $(<0.2 \mathrm{mg} / \mathrm{ml})$ is perhaps under the regulation of endogenous auxin whose levels depend on the cell density. The axenic cell cultures have been examined for the presence of IAA and their ability to metabolize labelled tryptophan into IAA (Jayaswal and Johri, 1985). The endogenous level of IAA was observed to be approximately 1.9 and $5.0 \mu \mathrm{g} / \mathrm{kg}$ fresh weight in chloronema and predominantly caulonema cells respectively. A similar level of IAA, $2.1 \mathrm{ng} / \mathrm{gm}$ fresh weight of gametophytictissue (predominantly chloronema) has also been found in $P$. patens (Ashton et al., 1985). Tryptophan is metabolized into IAA by intact chloronema cells and by the cell free homogenates. More IAA accumulated when homogenates from cells pre-grown at low cell densities $(<0.5 \mathrm{mg} / \mathrm{ml})$ were used as compared to those at high cell densities $(>0.5 \mathrm{mg} / \mathrm{ml})$. Since the activities of peroxidase and IAA-oxidase are known to be high at high cell densities, the lack of accumulation of IAA at high densities can be attributed to a high level of IAA-oxidizing enzymes (Sharma et al., 1979; Jayaswal and Johri, 1985). These results are consistent with a possible relationship between IAA accumulation and caulonema differentiation. In P. patens, a constitutive expression of homologues of the Arabidopsis genes which regulate auxin biosynthesis, led to elevated auxin levels and increased caulonema/chloronema ratio (Eklund et al., 2010). In this moss, auxin has been shown to promote caulonema differentiation by positively regulating two basic helix-loop-helix type of transcription factor genes namely ROOT HAIR DEFECTIVE SIX-LIKE1 (PpRSL1) and PpRSL2 (Jang and Dolan, 2011). It is conceivable that genes homologous to these transcription factors are also involved in the regulation of caulonema differentiation in the moss Funaria.

\section{Is there a minimal number of chloronema cells before $2^{\circ}$ caulonema differentiate?}

In Funaria, a minimal number of chloronema cells seem to be formed before a caulonema apical cell differentiates (Johri, 1978). Three sub-cellular events culminating in caulonema formation are distinguishable. These are: partitioning of chloroplasts, formation cells with reduced diameter, and orientation of mitotic spindle preceding the formation of first oblique septa in a cellular aggregate. In addition to these, the frequency of cell division in the caulonema apical cell and competence of caulonema to respond to cytokinins must also be regulated. In suspension cultures grown in continuous light, on an average three caulonema cells get added after 24 hours.

In liquid cultures using inoculum comprising of various cellular aggregates, the first caulonema initial is formed by the time an ag-
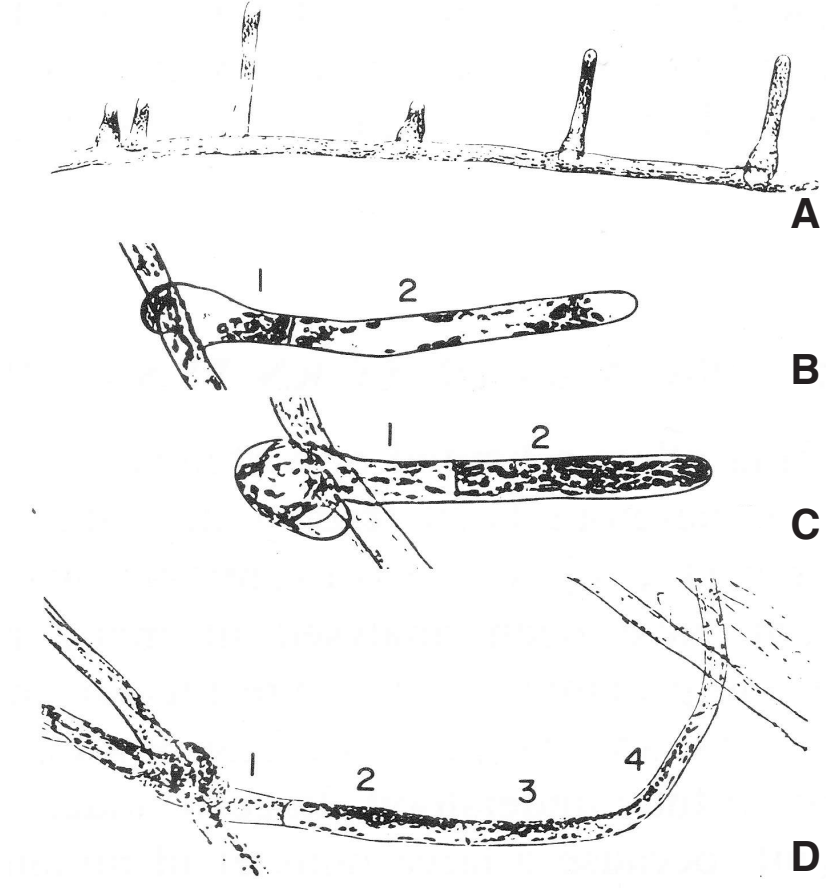

Fig. 2. Redifferentiation of $2^{\circ}$ caulonema from dedifferentiated bud initials in the mutant pg-1. (A) Elongation of the bud initial forming the side branch initials. (B,C) The apical cell divides and chloroplasts divide in this cell leading to the organization of a chloronema apical cell. (D) The apical cell divides and after producing several chloronema cells (marked 1-4), caulonema cells are produced. Originally published in Johri, MM (1988). Development and differentiation in plants. Bioscience Reports. 8: 553-564.

gregate has 4- 9 cells. The mutant $p g$ - 1 of $F$. hygrometricaillustrates this feature (Johri, 1988). In the absence of exogenous auxin, this mutant produces predominantly caulonema filaments (>65\%) and also no gametophores are formed (Handa and Johri, 1979). The spontaneously arising bud initials were observed to dedifferentiate into chloronema and then redifferentiate into caulonema (Fig. 2). A bud initial elongates, divides into two cells and the chloroplasts multiply in the tip cell leading to the organization of a typical chloronema apical cell. In no case the bud initials redifferentiated directly into caulonema. The dedifferentiated initials produced a few chloronema cells before redifferentiating into caulonema (Fig. 2). Sixty per cent of dedifferentiated initials produced $4 \pm 1$ chloronema cells, while the remaining produced up to 9 cells (Johri, 1988). The number of chloronema cells could not be reduced or eliminated upon growing the mutant with auxin. Whether a cell counting or a distance measurement or build-up of an endogenous factor above a certain threshold is required is not known.

\section{Bulk medium pH and caulonema and rhizoid differentiation}

The relationship between inoculum size, medium $\mathrm{pH}$ and auxin has been extensively investigated (Johri and D Souza, 1990). In a batch culture using $8.4 \mathrm{mM}$ nitrate as the sole $\mathrm{N}$-source; as IAAenhanced caulonema differentiate, the bulk medium $\mathrm{pH}$ changes from an initial value of $5.1 \pm 0.1$ to $6.1 \pm 0.2$. This $\mathrm{pH}$ range during differentiation is similar to that found for other auxin-induced 
responses. In order to further optimize the differentiation, cells were cultured in the medium buffered at $\mathrm{pH} 5-7$. At $\mathrm{pH} 5$, the response to IAA is slightly delayed, but most unexpectedly caulonema differentiate even in the control cultures after a lag of 6 \pm 1 days. The differentiation is independent of inoculum size up to $2.5 \mathrm{mg} / \mathrm{ml}$ and lag period of 6 days at a cell density of 0.05 $\mathrm{mg} / \mathrm{ml}$ is reduced to $3-4$ days at a cell density of $1 \mathrm{mg} / \mathrm{ml}$ (Johri and D'Souza, 1990).

In the medium buffered at $\mathrm{pH} 6$ or 7 , whereas the $1^{\circ} \mathrm{caulonema}$ differentiate normally, but the formation of $2^{\circ}$ caulonema is greatly reduced. The observed pattern of caulonema differentiation in auxin-free medium at $\mathrm{pH}$ 5-6 suggests that the transport of IAA occurs as passive diffusion of undissociated molecules. But the possibility of a carrier-mediated auxin influx which has an optimum of about $\mathrm{pH} 6$ and half saturated at 1-5 $\mu \mathrm{M}$ auxin (Rubery and Sheldrake, 1974), is not ruled out. In protonema grown in low light there is strong evidence for the presence of auxin influx and efflux carriers (Rose et al., 1983). As IAA accumulation in the protonema is strongly $\mathrm{pH}$ dependent, being greatly enhanced at $\mathrm{pH} 4$ as compared to that at $\mathrm{pH} 7.6$ (Rose et al., 1983), the low proportion of caulonema formed at $\mathrm{pH} 6$ or 7 in the IAA-medium could be due to reduced uptake and accumulation of auxin. The differentiation of caulonema in auxin-free medium buffered at $\mathrm{pH}$ 5 could conceivably be either due to a change of the sensitivity to endogenous IAA or due to a build-up of auxin (intracellular or in the medium) above a threshold during the lag period. Even if the auxin synthesized by proliferating chloronema and $1^{\circ}$ caulonema diffused into the medium, at $\mathrm{pH} 5$ a passive diffusion back into the cells would be favored. The presence of IAA in Funaria liquid cultures has not been investigated but IAA export has been found in $P$. patens. In this moss, PIN-dependent intercellular auxin transport is operational and the cultivation medium of liquid cultures shows an auxin level of about $700 \mathrm{pg} \mathrm{IAA/g}$ of medium (Viaene et al., 2014).

After 10 days as the liquid cell cultures became old, the socalled caulonema formed were unbranched, brown, with highly reduced number of chloroplasts, and diameter and did not form buds in response to cytokinins. In Funaria, mutants producing predominantly rhizoids with IAA have been isolated (Johri and D'Souza 1990). In the mutant 72, IAA-enhanced rhizoid production occurs at a concentration lower than that stimulating caulonema. In the presence of 0.6 to $10 \mu \mathrm{M}$ IAA, medium $\mathrm{pH}$ remained below 5.0, and most of the $2^{\circ}$ chloronema and caulonema were observed to redifferentiate into rhizoids with the result that the cultures showed $>80 \%$ rhizoids (Table 1 ). As already stated, auxins also markedly stimulate the formation of rhizoids in the thalli of liverworts and at the base of moss plants. The filaments in the old cultures or in the mutants are remarkably similar to the multicellular rhizoids which arise at the base of gametophores. Because of the lack of cytokinin response, we have referred them as protonemal rhizoids. Whether to consider them as atypical caulonema or protonemal rhizoids is a difficult question to answer. In fact rhizoids being similar to caulonema have been proposed to be products of same differentiation process (Knoop, 1984). Interestingly, the same two transcription factor genes (PpRSL1 and PpRSL2) in $P$. patens have been found to be adequate for the formation of caulonema in the protonema and rhizoids arising at the base of gametophytes (Jang and Dolan, 2011; Jang et al., 2011); furthermore, auxin positively regulates the expression of
TABLE 1

\section{CAULONEMA AND RHIZOID DIFFERENTIATION WITH IAA IN THE MUTANT 72 OF F. HYGROMETRICA}

\begin{tabular}{cccccc}
$\begin{array}{c}\text { IAA } \\
(\boldsymbol{\mu M})\end{array}$ & $\begin{array}{c}\text { Fresh weight } \\
\% \text { of control }\end{array}$ & $\begin{array}{c}\text { Medium } \\
\mathbf{p H}\end{array}$ & $\begin{array}{c}\text { Chloronema } \\
\%\end{array}$ & $\begin{array}{c}\text { Caulonema } \\
\%\end{array}$ & $\begin{array}{c}\text { Rhizoids } \\
\%\end{array}$ \\
\hline 0 & 100 & 6.66 & 93 & 7 & 0 \\
0.01 & 81 & 6.39 & 93 & 7 & 0 \\
0.10 & 62 & 4.93 & 55 & 5 & 40 \\
0.30 & 56 & 4.62 & 23 & 22 & 50 \\
0.60 & 62 & 4.74 & 18 & $<10^{\mathrm{b}}$ & Predominant \\
1.0 & 61 & 4.58 & 8 & $<10^{\mathrm{b}}$ & Predominant \\
2.0 & 49 & 4.56 & $<10^{\mathrm{b}}$ & $<15^{\mathrm{b}}$ & Predominant \\
5.0 & 53 & 4.61 & $<10^{\mathrm{b}}$ & $<15^{\mathrm{b}}$ & Predominant \\
10.0 & 43 & 4.62 & $<10^{\mathrm{b}}$ & $<10^{\mathrm{b}}$ & Predominant \\
\hline
\end{tabular}

a Cells were cultured in MMG at an inoculum density of $0.05 \mathrm{mg} \mathrm{ml}^{-1}$. Responses were scored 6 days after culture. The $\mathrm{pH}$ of the medium in IAA treated cultures of the mutant remains below 5 . Predominant implies $>80 \%$ filaments. The mutant was isolated following mutagenesis with nitrosomethyl urea and was identified directly on the basis of altered morphology on IAA-containing medium. Fresh weight in control was $0.68 \mathrm{~g} \mathrm{l}^{-1}$

${ }^{\mathrm{b}}$ Most of the $2^{\circ}$ chloronema and caulonema redifferentiated into rhizoids.

Reproduced with Permission from Johri and D'Souza, 1990. @ Springer-Verlag, Berlin Heidelberg 1990

these genes to promote either caulonema or rhizoid differentiation.

\section{Caulonema and rhizoid differentiation in nitrogen- limited medium}

In Funaria, following spore germination on phosphate- or nitrate-deficient medium, there is an excessive formation of rhizoids and a retarded development of chloronema (Schoene, 1906). Rhizoids being similar to caulonema, have been considered as products of the same differentiation process (Knoop, 1984). These observations suggest that there must be a relationship between starvation and caulonema and or rhizoid differentiation. Our studies show that there is a gradient of nutrients around a 10-12-day-old protonema growing on the agar surface. At various distances from the protonema, the analysis showed that the levels of nitrate, phosphate and glucose are respectively $63 \%, 42 \%$ and $51 \%$ of that present farthest away from the protonema. Thus, the protonema growing on the agar surface form caulonema spontaneously and also experience a nutrient-limited environment. Daily transfers of protonema on to the fresh medium (so as to prevent it from nutrient limitation), reduces drastically the production of caulonema. This reduction was not due to the act of transfer per se, because on nitrate-limited medium $(0.5 \mathrm{mM}$ nitrate as compared to the normal level of $8.4 \mathrm{mM}$ ) the level of caulonema differentiation is same in the transferred and untransferred protonema (Johri, 1978). Results with phosphate- or sulfate-limited medium are similar to those obtained with nitrate-limited medium. Thus a limitation of nutrients (e.g., partial starvation) enhances caulonema differentiation in protonema growing on agar surface.

Similar enhancement also occurs in auxin-free nitrate-limited liquid medium. In a medium containing 0.5 to $2.5 \mathrm{mM}$ nitrate, both caulonema and rhizoids are formed and their formation is independent of inoculum size between 0.05 and $2.5 \mathrm{mg} / \mathrm{ml}$ (Johri and D Souza, 1990). The cells cultured at a high cell density (0.5 $\mathrm{mg} / \mathrm{ml}$ ) in the low nitrate medium (LNM, nitrate $1.5 \mathrm{mM}$ ) responded to $1 \mu \mathrm{M}$ IAA, while cells in the medium with adequate nitrate (MMG, nitrate $8.4 \mathrm{mM}$ ) responded very slightly to $3 \mu \mathrm{M}$ IAA. Upon buffering these media at $\mathrm{pH} 5$, cells responded to lower auxin 
concentration and caulonema differentiated at 10-30 nM IAA. The cells grown in LNM or MMG respond to more or less same range of IAA concentrations, but the proportion of caulonema and rhizoids was always more in LNM. In these experiments caulonema differentiation with IAA was scored 3 days after transfer of cells because later on the caulonema differentiate even in the control flasks. Unlike the low level of differentiation with IAA in MMG buffered at $\mathrm{pH} 6$ or 7 , about $35-40 \%$ caulonema and rhizoids differentiated even at higher densities in the LNM buffered at same $\mathrm{pH}$ values. Therefore, under sub-optimal growth conditions, cells seem to acquire enhanced sensitivity to respond to exogenous or endogenous auxin. An enhanced caulonema differentiation by 1-10 nM IAA, occurs in the protonema growth in low light (Lehnert and Bopp, 1983). It is suggested that under the nutrient-limited conditions, the enhanced sensitivity primes the cells to respond to low endogenous auxin levels and culminates in the expression of genes involved in caulonema differentiation. Based on this reasoning, the formation of caulonema spontaneously on the solid medium can be explained. These results at physiological level suggest very useful and tractable leads to understand auxin perception and signal transduction in moss.

\section{Classical auxin-binding protein (ABP1) homologues in Funaria lack ER-retention sequences}

Auxin inhibition of chloronema and enhancement of caulonema are both antagonized by anti-auxin $p$-chlororophenoxyisobutyric acid (PCIB). PCIB inhibits basipetal auxin transport in Funaria rhizoids (Rose and Bopp. 1983) and also competes with IAA for IAA binding sites (reviewed in Johri and D'Souza, 1990). In Arabidopsis roots, PCIB impairs auxin-signaling pathway by reducing the stability of auxin/indole-3-acetic acid (Aux/IAA) protein (Oono et al., 2003). The foregoing arguments show that both basipetal transport and IAA-binding sites are involved in auxin responses at the level of chloronema and caulonema. In order to find the proteins involved in auxin binding, we have examined for the presence of classical auxin-binding protein (ABP1) homologues in F. hygrometrica (Panigrahi et al., 2009). Microsomal membranes from the protonema (chloronema cells harvested at a cell density of $4-5 \mathrm{mg} / \mathrm{ml}$ ) showed specific IAA-binding sites, estimated to be 3-5 pmol/mg protein with an apparent dissociation constant $\left(K_{d}\right)$ between 3 and $5 \mu \mathrm{M}$. Using the anti-ABP1 antiserum, the canonical ER-localized 22-24 kDa ABP1 was detected in Zea mays (used as positive control), but not in F. hygrometrica. Instead polypeptides of 31-33 and 46 kDa were detected both in moss and corn. In the moss, these polypeptides were localized in the microsomal membrane fraction and were photo-affinity labelled with 5-azido-[ $\left.7^{3} \mathrm{H}\right]-I A A$. Unlike the corn ABP1, the moss ABPs seem to be different and are unlikely to be ER-localised. The immunological methods showed the absence of KDEL or HDEL ER-retention sequence. Likewise, the analysis of ABP1 sequence in the genome of Physcomitrella patens and Ceratodon purpureus also failed to show the presence of signal sequences associated with the retention in ER. These data strongly suggest that the Funaria ABPs have a distinct novel signature and may not be ER-localized. Panigrahi et al., (2009) have further suggested that the ER-retention sequences of ABP1 could have evolved later during the evolution of higher plants coincident with the evolution of a more elaborate auxin signaling.

\section{Mutants with altered differentiation pattern in mosses}

Several mutants with altered differentiation patterns have been isolated and analyzed at physiological level but the success with $F$. hygrometrica has been rather limited and most of this information has been reviewed by Bhatla (1994), here only those relevant to caulonema formation will be discussed. The pale green -1 (pg-1) mutant of Funaria produces spontaneously far more caulonema than chloronema; it responds to 3'5'-cyclic AMP and chloronema can be restored substantively (Handa and Johri, 1979). It does not respond to cytokinins and the effect of TIBA (2,3,5-triiodobenzoic acid) or PCIB on chloronema formation was not tested. As already discussed, the bud initials dedifferentiated and after the formation of $4 \pm 1$ chloronema cells, the caulonema initials are formed (Johri, 1988 ). The NAR2 mutant ( $\alpha$-NAA resistant 2 ) is blocked in caulonema formation and can be normalized with auxin (Bhatla and Bopp, 1985). It showed enhanced IAA oxidase activity suggesting that the lack of caulonema is due to low level of endogenous auxin.

The protonema $P$. patens moss has turned out to be far more amenable to mutant isolation and genetic analysis as compared to Funaria. Genome sequences coding for auxin transport, perception and signaling have been identified in the genome of $P$. patens (Rensing et al., 2008). Several mutants have been analyzed at physiological level and found to have altered auxin homeostasis, blocked biosynthesis or perception. Some of these having a chloronema phenotype can be normalized by exogenous auxin. The emerging picture suggests that we are beginning to learn about auxin perception, role of auxin response factor genes and subsequent signal transduction events in mosses and these are turning out to be highly conserved and in many respects similar to that in the Arabidopsis. Several auxin resistant mutants have been shown to carry mutations in Aux/IAA transcriptional repressor genes (Ashton et al., 1979; Prigge et al., 2010). The P. patensAux/ IAA protein interacts with PpAFB moss homologues of Arabidopsis TIR1 and auxin signaling in chloronema cells is mediated through PpAFB proteins.

\section{Concluding remarks and some historical recollections}

The studies on moss protonema discussed here were carried out at TIFR in the pre-cyber era and until 1980 there were no laptop or desktop computers, internet or even an easy access to telephones. In that era doing science was more of an art, far more challenging and one could remain competitive only on the basis of original ideas (or unconventional ideas as several of my colleagues used to say) and by employing novel experimental strategies. The notion of unconventional idea is best illustrated by our successful demonstration of a high affinity 3',5'-cyclic AMP-PDE from the moss protonema (Sharma and Johri, 1983) by employing ammonium sulfate fractionation at alkaline $\mathrm{pH}$. The PDE from higher plants typically prefer 2',3'-cAMP as substrate, are optimally activity at acidic $\mathrm{pH}$ and are different from animal and bacterial PDEs which utilize 3', 5'-cAMP and exhibit maximum activity at basic $\mathrm{pH}$. The moss protonema showed both acidic and alkaline PDEs, At the crude extract stage, the PDE activity from Funaria exhibited a single $\mathrm{pH}$ optimum in the acidic range $(\mathrm{pH} 5.0-5.5)$, and therefore during purification PDE activity was monitored at $\mathrm{pH}$ 5.4. This led to a selective purification of the acid PDE which was $\mathrm{Zn}^{2+}$-dependent. Although the alkaline PDE survived this purifica- 
tion procedure but its yield was low (Sharma and Johri, 1982). We therefore characterized the PDE by monitoring the activity at $\mathrm{pH}$ 7.5 during purification (Sharma and Johri, 1983). These efforts led to the finding two new features: i) the high affinity 3',5'-cAMP specific moss protonemal PDE was $\mathrm{Ca}^{2+}$-dependent and ii) the alkaline PDE was converted partially into the acid PDE during the purification protocol. The interconversion of one molecular form of PDE to another has earlier been found in other tissues also (see Sharma and Johri, 1983). The moss 3',5'- cAMP specific PDE is remarkably similar to the mammalian $\mathrm{PDE}$ which are also $\mathrm{Ca}^{2+}$ dependent and exhibit a high affinity $K_{m}$ for the substrate.

Looking back, TIFR offered the most challenging academic ambience and a vibrant atmosphere with immense opportunities that were compatible with my own style of working and thinking. Like others at that time in early 1970s, I also thought that the best strategy for understanding the basic rules underlying cell differentiation, will be to focus on simple systems, such as mosses which are interesting in their own right. This expectation has been at least partially fulfilled and furthermore my studies were funded entirely by in-house Institutional funding. Only for participating in International Conferences abroad, funds had to be raised from various agencies. At no time I had to project the relevance of my studies to human need, human disease or biotechnology. This is quite a contrasting scenario as compared to the present situation where one has to project well in advance the applications or applied importance, very often even before having initiated the research or exploratory studies. Such a projection does not always work out as far as basic studies are concerned.

At present, with the advances in the analytical methods and ready availability of table top versions of instruments, and the genome sequences of two mosses, biology of mosses has become far more analytical, challenging and truly an exciting one. I wish to dedicate this paper to late Prof. Dr. Martin Bopp who passed away on August 30, 2018. I first met him almost fifty years ago in Aug 1969 at the XI International Botanical Congress at Seattle, Wash., USA; at that time I had no idea that in future Funaria protonema will be an experimental paradigm for my research investigations. Since 1973, it has been a very fruitful interaction with him during the International Conferences (Box 1) and during my visits to Heidelberg. It is nice to know that the auxin regulation of caulonema has become a part of basic knowledge in the field of moss biology. It is a satisfying feeling that our 1973 paper describing caulonema differentiation by auxin published in Nature New Biology, continues to be cited even after 45 years (Jang and Dolan, 2011; Zhao et al., 2018). It also provided the missing piece of information to

Fig. 3. Moss Biology Group at the Tata Institute of Fundamental Research (TIFR) in mid-1998. It often met for informal group meetings on the weekends. During one such meeting Obaid Siddiqi (Chair, Department of Biological Sciences) and James Watson walked in unannounced. Obaid left Watson to interact with students and this photograph was taken at that time. Watson had visited India in the summer of 1998 in connection with the Human Genome Project. From left to right: Doyel Mitra; Kishore Panigrahi; M. M. Johri; James Watson; Jacinta S. D'Souza and Suvarna Ainapure. (Photo courtesy of Kishore Panigrahi).
Box 1. Historically speaking, the studies on caulonema differentiation were first presented publicly at the $8^{\text {th }}$ International Conference on Plant Growth Substances held at Tokyo in Aug. 1973. The session was chaired by Prof. P.E. Pilet from the University of Lausanne, Switzerland. The moment I stated that "I wish to describe a new response to auxin at cellular level using the protonema of the moss Funaria, as an experimental system" there was total silence and everybody turned attentive. By that time most of auxin responses at cell, tissue or organ levels had already been discovered and our findings came as a total surprise and new to everybody. Prof. Martin Bopp (Botanical Institute, University of Heidelberg, Germany), a leading authority on moss development, was among the audience and complimented me after the talk. Upon returning to Heidelberg his group repeated my work and Bopp wrote to me that they have confirmed my findings and he further suggested that we should consider publishing it jointly. I replied him that our paper is already accepted for publication; it was submitted on March 27, revised August 6 and published Oct.17, 1973 (Johri and Desai 1973, Auxin regulation of caulonema formation in moss protonema. Nature New Biology 245:223-224).

explain the origin of auxin response at the level of bryophytes in the green cell lineage or the early diverging streptophyhte plants. It is still not clear if the native auxin regulates cellular responses in green algae; efflux carriers which are insensitive to polar auxin transport inhibitors seem to be present in the thallus tips of Charophytes (see Cooke et al., 2002). Only future work will clarify the picture about green algae. Likewise, although pharmacological effects of auxin are known in liverworts for the past forty to fifty years, but still there is no unequivocal evidence that auxin acts as a native hormone in them. In mosses there is now unequivocal evidence for the native hormonal function of auxin and it should be relatively easier to understand the auxin action mechanism where it first evolved. Mosses seem to provide just that window of opportunity in the evolutionary time frame and in future one should expect rapid advances on that front.

In retrospect, our studies on caulonema differentiation have

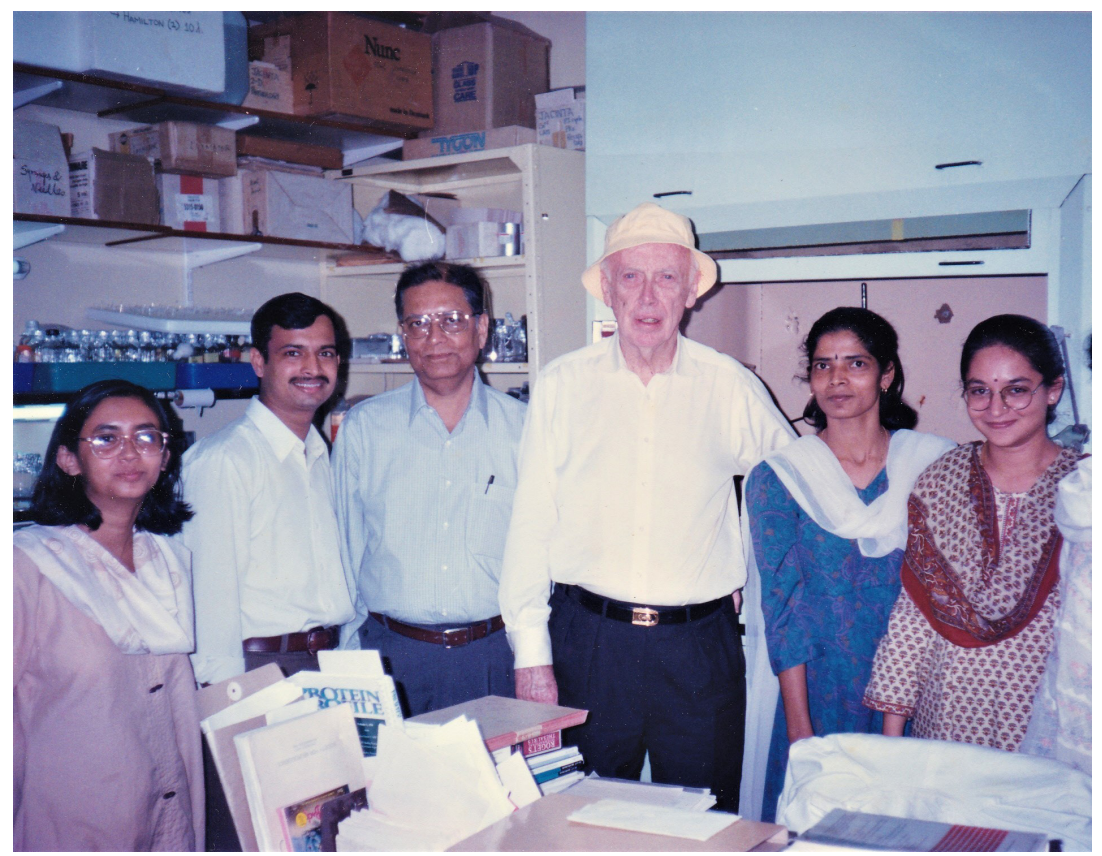


drawn a lot of attention. Bopp (1980) has termed it as an important discovery in the field of hormonal regulation of morphogenesis in Mosses. He writes "The clear definition of chloronema and caulonema as the two main stages in the development of the moss protonema by Sironval (26) ${ }^{\star}$ in 1947 established the mosses as a tool for morphogenetic studies. Subsequent important discoveries for this purpose were the findings by Gorton and Eakin (26)* that kinetin can induce bud formation in the protonema of Tortella caespitosa and the demonstration by Johri and Desai $(38)^{\star}$ that exogenous auxin induces the transition from chloronema to caulonema in a suspension culture of Funaria hygrometrica. Previously only an inhibitory effect of auxin on protonemal growth was well documented (5)." The asterisks ( ${ }^{*}$ ) have been introduced by the author of this article and the numbers 26, 38 and 5 refer to the serial numbers of references cited by Bopp (1980).

\section{Acknowledgments}

I am thankful to my students especially to Dr. Jacinta S. D'Souza, Dr. Kishore Panigrahi and Dr. Shobhona Sharma. They were associated with various phases of the studies described here. A major activity at the Department of Biological Sciences were the ICRO Courses, winter schools and lectures by distinguished scientists. During these events, there were plenty of opportunities for the academic members and students to interact with the visitors (Fig. 3). It is also a pleasure to acknowledge several novel suggestions from my colleagues at the Department of Biological Sciences. The academic ambience of the Department and a very fruitful interaction with colleagues were among the driving forces to think differently. It made it possible to build my own brand. Ultimately it was the success and recognition from the scientific community that mattered the most.

\section{References}

ASHTON N W, GRIMSLEY, N H and COVE D J (1979). Analysis of gametophytic development in the moss, Physcomitrella patens, using auxin and cytokinin resistant mutants. Planta 144: 427-435.

ASHTON N W, SCHULZE A, HALL P and BANDURSKI R S (1985). Estimation of indole-3-acetic acid in gametophytes of the moss, Physcomitrella patens. Planta 164: $142-144$.

BHATLA S C (1994). Moss Protonema Differentiation. Research Studies Press Ltd., Taunton, Somerset, England. John Wiley \& Sons Inc.

BHATLA S C and BOPP M (1985). The hormonal regulation of protonema development in mosses. III: Auxin - resistant mutants of the moss Funaria hygrometrica Hedw. J. Plant Physiol. 120: 233-243.

BOPP M (1980). The hormonal regulation of morphogenesis in mosses. In: Plant Growth Substances 1979. (Ed. F. Skoog). Springer-Verlag, Berlin. pp. 351-361.

BOPP M, QUADER H, THONI C, SAWIDIS T and SCHNEPF E (1991). Filament disruption in Funaria protonemata: formation and disintegration of Tmema cells. J. Plant Physiol. 137: 273-284

BRANDES $H$ and KENDE H (1968). Studies on cytokinin-controlled bud formation in moss protonema. Plant Physiol. 43: 827-837.

COOKE T J, POLI D B, SZTEIN E and COHEN J D (2002). Evolutionary patterns in auxin action. Plant $\mathrm{Mol} \mathrm{BiO}$ 49: 319-338.

EKLUND D M, THELANDER M, LANDBERG K, STÅLDAL V, NILSSON A, JOHANSSON M, VALSECCHI I, PEDERSON E R, KOWALCZYK M, LJUNG K, et al., (2010). Homologues of the Arabidopsis Thaliana SHI/STY/ LRP1 genes control auxin biosynthesis and affect growth and development in the moss Physcomitrella patens. Development 137: 1275-1284.

GORTON B S and EAKIN R E (1957). Development of the gametophyte in the moss Tortella caespitosa. Bot Gaz 119: 31-38.

HANDA A K and JOHRI M M (1979). Involvement of cyclic adenosine-3', 5'-monophosphate in chloronema differentiation in protonema cultures of Funaria hygrometrica. Planta 114: 317-324.

ISHIKAWA M, MURATA T, SATO Y, NISHIYAMA T, HIWATASHI Y, IMAI A, KIMURA
M, SUGIMOTO N, AKITA A, OGURI Y, FRIEDMAN W E, HASEBE $M$ and KUBO M (2011). (2011) Physcomitrella cyclin-dependent kinase A links cell cycle reactivation to other cellular changes during reprogramming of leaf cell. Plant Cell 23: 2924-2938.

JANG G and DOLAN L (2011). Auxin promotes the transition from chloronema to caulonema in moss protonema by positively regulating PpRSL1 and PpRSL2 in Physcomitrella patens. New Phytol 192:319-327.

JANG G, YI K, PIRES N D, MENAND B and DOLAN L. (2011). RSL genes are sufficient for rhizoid system development in early diverging land plants. Development 138: 2273-2281.

JAYASWAL R K and JOHRI M M (1985). Occurrence and biosynthesis of auxin in protonema of the moss Funaria hygrometrica. Phytochemistry 24: 1211-1214.

JOHRI M M (1974). Differentiation of caulonema cells by auxins in suspension cultures of Funaria hygrometrica. In Plant Growth Substances 1973 (Ed. S Tamura) Hirokawa Publishing Company, Tokyo, Japan pp. 925-933.

JOHRI M M (1975). The protonema of Funaria hygrometrica as a system for studying cell differentiation. In Form, Structure and Function in Plants (Eds H Y Mohan Ram, J J Shah and C K Shah) Sarita Prakashan, Meerut, India. pp. 116-124.

JOHRI M M (1978). Regulation of cell differentiation and morphogenesis in lower plants. In Frontiers of Plant Tissue Culture 1978 (Ed. T A Thorpe) IAPTC, University of Calgary Offset Printing Services, Calgary, Alberta, Canada. pp. 27-36.

JOHRI M M (1988). Development and differentiation in plants. Biosci. Rep. 8: 553-564.

JOHRI M M (2004). Possible origin of hormonal regulation in green plants. Proc. Indian Nat Sci Acad B29: 335-365.

JOHRI M M and DESAI S (1973) Auxin regulation of caulonema formation in moss protonema. Nature New Biol. 245: 223-224.

JOHRI, M M and D'SOUZA J S (1990). Auxin regulation of cell differentiation in moss protonema. In Plant Growth Substances 1988 (Eds. R P Pharis, and S B Rood) Springer-Verlag, Berlin, Heidelberg. pp. 407-418.

KAUL K N, MITRA G C and TRIPATHI B K (1962). Response of Marchantia in aseptic culture to well-known auxins and antiauxins. Ann Bot 26:447-466.

KNOOP B (1984). Development in Bryophytes. In The Experimental Biology of Bryophytes (Eds A F Dyer and J G Ducket) Academic Press, London. pp. 143-176.

KOFUJI R and HASEBE M (2014). Eight types of stem cells in the life cycle of the moss Physcomitrella patens. Curr Opin Plant Biol 17:13-21.

LEHNERT B and Bopp M (1983). The hormonal regulation of protonema development in mosses. I. Auxin-cytokinin interaction. Z Pflanzenphysiol 110:379-391.

OONO Y, OOURA C, RAHMAN A, ASPURIA E T, HAYASHI K, TANAKA A and UCHIMIYA H (2003). p-Chlorophenoxyisobutyric acid impairs auxin response in Arabidopsis root. Plant Physiol 133:1135- 1147.

PANIGRAHI K C S, PANIGRAHY M, VERVLIET-SCHEEBAUM M, LANG D, RESKI $R$ and JOHRI M M (2009). Auxin-binding proteins without KDEL sequence in the moss Funaria hygrometrica. Plant Cell Rep. 28:1747-1758.

PRIGGE M J, LAVY M, ASHTON N W and ESTELLE M (2010). Physcomitrella patens auxin-resistant mutants affect conserved elements of an auxin-signaling pathway. Curr. Biol. 20: 1907-1912.

RENSING S, LANG D, ZIMMER A D, TERRY A, SALAMOV A, SHAPIRO H, NISHIYAMA T, PERROUD P F, LINDQUIST E A, KAMISUGI Y, et al., (2008). The Physcomitrella genome reveals evolutionary insights into the conquest of land by plants. Science 319: 64-69.

ROHWER F and BOPP M (1985). Ethylene synthesis in moss protonema. J Plant Physiol 117: 331-338.

ROSE S and BOPP M (1983). Uptake and polar transport of indoleacetic acid in moss rhizoid. Physiol Plant 58:57-61.

ROSE S, RUBERY P H and BOPP M (1983). The mechanism of auxin uptake and accumulation in moss protonema. Physiol Plant 66:344-348.

RUBERY P H and SHELDRAKE A R (1974). Carrier-mediated auxin transport. Planta 118:101-121.

SAKAKIBARAK, NISHIYAMA T, SUMIKAWAN, KOFUJI R, MURATA T and HASEBE $M$ (2003). Involvement of auxin and a homeodomain-leucine zipper I gene in rhizoid development of the moss Physcomitrella patens. Development 130: 4835-4846.

SATO Y, SUGIMOTO N, HIRAI T, IMAI A, KUBO M, HIWATASHI Y, NISHIYAMA T and HASEBE M (2017). Cells reprogramming to stem cells inhibit the reprogramming of adjacent cells in the moss. Physcomitrella patens. Sci. Rep. 7: 1909. 
SCHOENE K (1906). Beitrige zur Kenntnis der Keimung der Laubmoos-sporen und zur Biologie der Laubmoosrhizoiden. Flora (Jena) 96: 276 - 321

SHARMA S and JOHRI M M (1982). Partial purification and characterization of cyclic AMP phosphodiesterases from Funaria hygrometrica. Arch Biochem Biophys. 217:87-97.

SHARMA S. and JOHRI M M (1983). Cyclic AMP phosphodiesterases of Funaria hygrometrica. Phytochem. 22: 2715-2717.

SHARMAS, JAYASWALRK and JOHRI M M (1979). Cell density- dependent changes in the metabolism of chloronema cell cultures. I. Relationship between cell density and enzymic activities. Plant Physiol. 64:154-158.
SIRONVAL C (1947). Experiences sur les stages de developpement de la forme filamenteuse en culture de Funaria hygrometrica L. Bull Soc Bot Belg 79:48-78.

VIAENET, LANDBERG K, THELANDER M, MEDVECKAE, PEDERSON E, FERARU E, COOPER E D, KARIMI M, DELWICHE C F, LJUNG K, GEISLER M, SUNDBERG E, and JIŘí FRIML J (2014). Directional auxin transport mechanisms in early diverging land plants. Curr Biol 24: 2786-2791.

ZHAO M. LI Q, CHEN Z, LV Q, BAO F, WANG X and HE, Y (2018). Regulatory mechanism of $\mathrm{ABA}$ and $\mathrm{ABI} 3$ on vegetative development in the moss Physcomitrella patens. Int J Mol Sci 19: 2728

\section{Further Related Reading, published previously in the Int. J. Dev. Biol.}

Balance between cell division and differentiation during plant development

Elena Ramirez-Parra, Bénédicte Desvoyes and Crisanto Gutierrez

Int. J. Dev. Biol. (2005) 49: 467 - 477

doi: $10.1387 / \mathrm{ijdb} .052001 \mathrm{er}$

Instructive roles for hormones in plant development

David Alabadí, Miguel A. Blázquez, Juan Carbonell, Cristina Ferrándiz and Miguel A.

Pérez-Amador

Int. J. Dev. Biol. (2009) 53: 1597 - 1608

doi: $10.1387 / \mathrm{ijdb} .072423 \mathrm{da}$

Dynamical patterning modules in plant development and evolution

Valeria Hernández-Hernández, Karl J. Niklas, Stuart A. Newman and Mariana Benítez

Int. J. Dev. Biol. (2012) 56: 661 - 674

doi: $10.1387 / \mathrm{ijdb} .120027 \mathrm{mb}$

A "Brief History" of Developmental Biology in Israel

Dalit Sela-Donenfeld and Dale Frank

Int. J. Dev. Biol. (2017) 61: 115 - 120

doi: $10.1387 / \mathrm{ijdb} .170050 \mathrm{df}$

Gene network analysis in plant development by genomic technologies

Frank Wellmer and José Luis Riechmann

Int. J. Dev. Biol. (2005) 49: 745 - 759

doi: $10.1387 / \mathrm{ijdb} .051991 \mathrm{fw}$
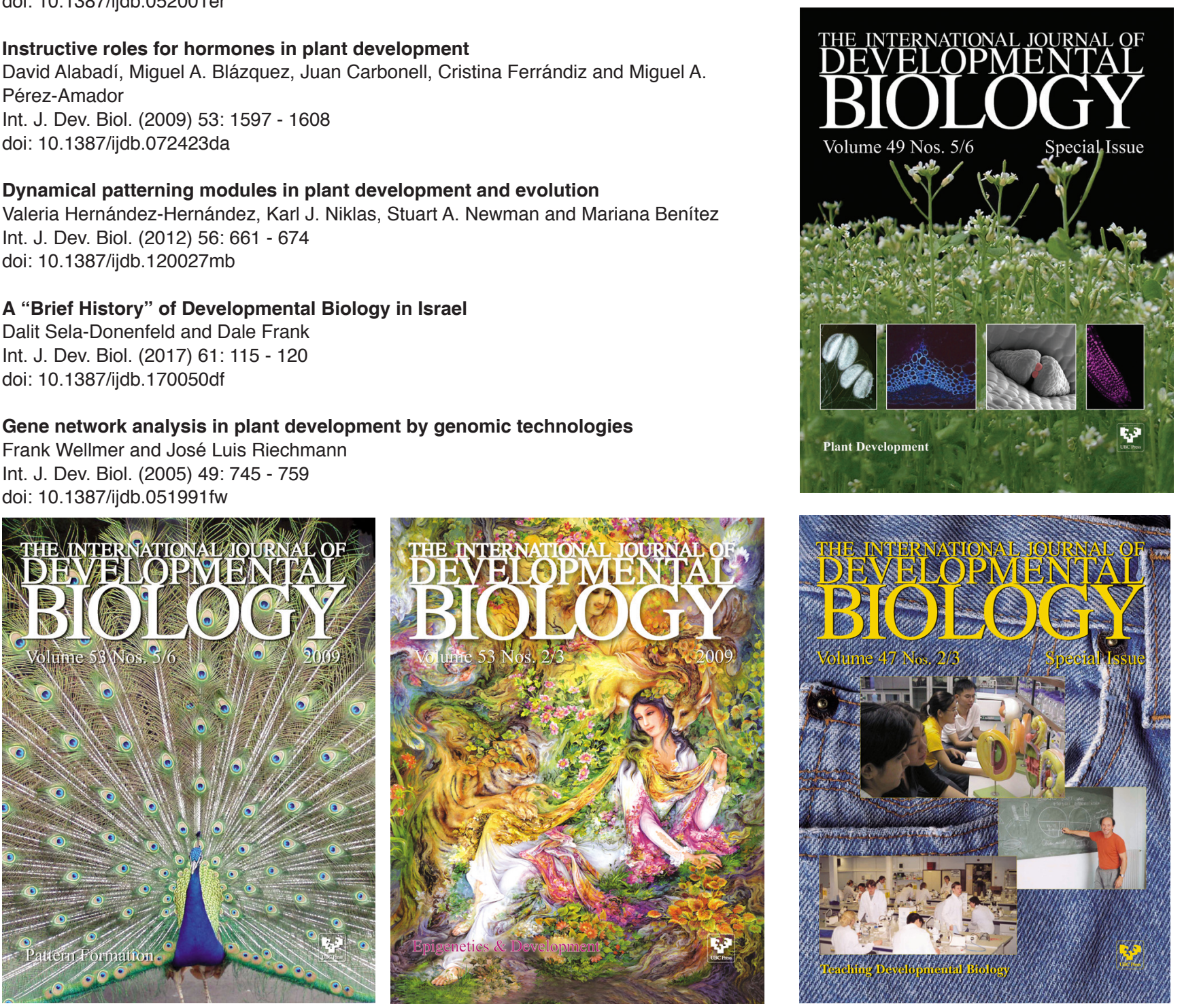